\title{
Educación Continua: construcción del conocimiento y estrategias educativas para las enfermeras de oncología
}

\author{
Continuing Education: construction of knowledge and educational \\ strategies by oncology nurses
}

\section{Educação Permanente: construção de saberes e estratégias educativas por enfermeiros oncológicos*}

\section{Fabiana Cristina dos Santos ${ }^{1}$, Ana Maria Laus ${ }^{2}$, Andrea Bernardes ${ }^{3} \&$ Silvia Helena Henriques Camelo ${ }^{4}$}

\footnotetext{
* Este artigo foi extraído do projeto financiado pela Fundação de Amparo à Pesquisa do Estado de São Paulo- FAPESP, Brasil- Número do processo: 2013/09203-1.
${ }^{1}$ Enfermeira. Mestre em Ciências pelo Programa de Pós-Graduação em Enfermagem Fundamental da Escola de Enfermagem de Ribeirão Preto- Universidade de São Paulo. Ribeirão Preto. São Paulo. Brasil. E-mail: fabiana.santos@usp.br

${ }^{2}$ Professora Associada, Doutora do Departamento de Enfermagem Geral e Especializada da Escola de Enfermagem de Ribeirão Preto- Universidade de São Paulo. Ribeirão Preto.

São Paulo. Brasil. E-mail: analaus@eerp.usp.br

${ }^{3}$ Professora Associada, Doutora do Departamento de Enfermagem Geral e Especializada da Escola de Enfermagem de Ribeirão Preto- Universidade de São Paulo. Ribeirão Preto. São Paulo. Brasil. E-mail: andreab@eerp.usp.br

${ }^{4}$ Professora, Doutora do Departamento de Enfermagem Geral e Especializada da Escola de Enfermagem de Ribeirão Preto- Universidade de São Paulo. Ribeirão Preto. São Paulo. Brasil. E-mail: shcamelo@eerp.usp.br
}

Cómo citar este artículo en edición digital: Santos, F.C., Laus, A.M., Bernardes, A., \& Camelo, S.H.H. (2018). Educación Continua: construcción del conocimiento y estrategias educativas para las enfermeras de oncología. Cultura de los

Cuidados (Edición digital), 22(51). Recuperado de < http://dx.doi.org/10.14198/cuid.2018.51.03>

Correspondencia: Fabiana Cristina dos Santos.Endereço: Avenida Bandeirantes, 3900 - Campus Universitário- Monte Alegre - Ribeirão Preto- São Paulo- Brasil- CEP: 14040-902.

Correo electrónico: fabiana.santos@usp.br Recibido: 09/12/2017; Aceptado: 07/04/2018

\begin{abstract}
Objective: Characterize the permanent training strategies developed by oncology nurses working in hospitals. Methodology: This is an exploratory study, a qualitative approach, and the methodological design the multiple case study. This research was performed in Oncology Units of two Teaching Hospitals and 30 nurses
\end{abstract}

participated. To collect data used semi-structured interviews and data analysis was performed using thematic analysis. Results: Strategies of education provided by the hospitals are pre-employment training, courses and scientific meetings, on various subjects, not being reported specific 
preparation in the area of oncology causing the professionals in these units, seek for specialized institutions in order to meet their professional development as necessary for his performance skills. As the strategies of education performed by nurses highlights the practical teaching from the routines of the service. Conclusion: This study will help nurses reflect on their practice considering the comprehensiveness of care provided to cancer patients, defining new modes, mechanisms and instruments for in-service training. Thus as institutions favor the training of these professionals using updated teaching strategies in this sector.

Keywords: Education, nursing, oncology service hospital, nurses, strategies.

\section{RESUMEN}

Objetivo: Caracterizar las estrategias de aprendizaje permanente desarrolladas por las enfermeras que trabajan en hospitales oncológicos. Metodología: Se trata de un estudio exploratorio de abordaje cualitativo, con el diseño metodológico del estudio de casos múltiples. Esta investigación se llevó a cabo en las Unidades de Oncología del participaron dos hospitales universitarios y 30 enfermeras. Para recoger los datos que hemos utilizado entrevistas semiestruturadas y análisis de datos se realizó mediante el análisis temático. Resultados: Estrategias de educación proporcionados por los hospitales son la formación de admisión, cursos y eventos científicos sobre diversos temas, no se informa preparación específica en oncología, por lo que los profesionales de estas unidades buscan por instituciones especializadas a cumplir con su desarrollo profesional como las habilidades necesarias para su funcionamiento. En cuanto a las estrategias de educación desarrollados por enfermeras resaltar la enseñanza práctica de las rutinas de servicio. Conclusión: Este estudio ayudará a las enfermeras a reflexionar sobre su práctica teniendo en cuenta la atención integral a pacientes con cáncer, la definición de nuevos procedimientos, mecanismos e instrumentos para la formación en el empleo. A medida que las instituciones favorecen la formación de estos profesionales que utilizan estrategias de enseñanza actualizada en este sector.

Palabras clave: Educación en enfermería, servicio de oncología en hospital, enfermeros, estrategias.

\section{RESUMO}

Objetivo: Caracterizar as estratégias de educação permanente desenvolvidas pelos enfermeiros atuantes em unidades hospitalares oncológicas. Metodologia: Trata-se de um estudo exploratório, de abordagem qualitativa, tendo como desenho metodológico o estudo de caso múltiplo. Esta investigação foi realizada em Unidades Oncológicas de dois Hospitais de Ensino e participaram 30 enfermeiros. Para a coleta de dados utilizamos entrevistas semiestruturadas e a análise dos dados foi realizada por meio da análise temática. Resultados: Estratégias de educação fornecidas pelas instituições hospitalares são os treinamentos admissionais, cursos e eventos científicos, sobre assuntos diversos, não sendo relatado preparo específico na área de oncologia, fazendo com que os profissionais destas unidades busquem por instituições especializadas a fim de suprir o seu desenvolvimento profissional quanto as competências necessárias para a sua atuação. Quanto às estratégias de educação desenvolvidas pelos enfermeiros destaca-se o ensino prático a partir das rotinas do serviço. Conclusão: Este estudo contribuirá para que os enfermeiros reflitam sobre sua práxis considerando a integralidade do cuidado prestado ao paciente oncológico, definindo novas modalidades, mecanismos e instrumentos para capacitação em serviço. Assim como as instituições favorecerem a capacitação destes profissionais utilizando estratégias de ensino atualizadas neste setor.

Palavras-chave: Educação em Enfermagem, serviço hospitalar de oncologia, enfermeiros, estratégias. 


\section{INTRODUÇÃO}

O trabalho de enfermeiros oncológicos consiste na prestação de cuidados à saúde envolvendo aspectos de ordem bio-psicosocio-espiritual, onde muitas vezes o paciente está fora da possibilidade de cura, e precisa ter, além de habilidade técnica para prestar cuidados físicos, sensibilidade nos aspectos psíquicos envolvidos, pautado na ética e na humanização (Salimena, Teixeira, Amorim, Paiva \& Melo, 2013). Assim, o enfermeiro necessita reavaliar constantemente o seu processo de trabalho, considerando as particularidades de cada paciente oncológico, em função das características específicas do adoecimento e responsabilidades que lhe são privativas, bem como, conhecimentos técnicocientíficos e habilidades no relacionamento interpessoal (Nascimento et al., 2012).

Nas unidades especializadas em oncologia devem-se buscar estratégias para a organização do trabalho de enfermagem, autonomia da equipe e assistência qualificada ao paciente oncológico, exigindo que saberes sejam construídos e aplicados constantemente, o que pode ocorrer por meio de atividades educacionais motivadoras e problematizadoras. Frente disso, a educação permanente representa uma habilidade de aprendizagem contínua, desenvolvida pelo sujeito durante a sua vida com o intuito de transformar a realidade, a partir do caráter educativo do próprio trabalho, tornando possível promover mudanças e transformações na perspectiva da integralidade da saúde (Ceccim, 2005). As ações de educação permanente tem como foco a solução de problemas na oferta dos serviços de saúde a uma dada população, incluídos aqui as instituições relacionadas à atenção oncológica (Silva, 2015). Neste sentido, a adoção de estratégias de educação permanente pelos enfermeiros oncológicos são determinantes para o planejamento e implementação do cuidado, visto que a formação realizada de modo eficaz refletirá na qualidade da assistência ao paciente.

Assim, podemos inferir que apesar de existirem algumas certezas, ainda não há consenso sobre todas as atividades de educação permanente desenvolvidas em unidades oncológicas. Todavia há que se estimular esta discussão, a fim de caracterizálas e mobilizar os enfermeiros para desenvolvê-las no ambiente de trabalho. Frente ao exposto formulamos os seguintes questionamentos: os enfermeiros desenvolvem estratégias de educação permanente para o seu aprimoramento e o de sua equipe? Existem dificuldades e/ou limitações enfrentadas por estes profissionais? A caracterização das atividades de educação permanente realizadas em unidades oncológicas contribuirá para a reflexão dos enfermeiros, gestores das organizações de saúde e centros formadores quanto à complexidade da área de atuação e necessidade de constante atualização da equipe de enfermagem, enquanto atores corresponsáveis na sua formação profissional. Assim este estudo teve o objetivo de caracterizar as estratégias de educação permanente implementadas pelas organizações de saúde e as desenvolvidas pelos enfermeiros durante sua atuação em unidades oncológicas.

\section{METODOLOGIA}

Trata-se de um estudo exploratório, de abordagem qualitativa dos dados. $\mathrm{O}$ delineamento metodológico da pesquisa é o estudo de caso, considerado um método de pesquisa de natureza empírica que investiga um fenômeno, geralmente contemporâneo, dentro de um contexto real, quando as fronteiras entre o fenômeno e o contexto em que ele se insere não são claramente definidas (Yin, 2015). Para este trabalho, definiu-se o estudo de caso múltiplo. Este tipo de estudo envolve a análise de dois ou mais casos; representa o caso decisivo para testar uma teoria bem formulada, seja para confirmá-la, seja para contestá-la, seja ainda para estender a teoria; ele representa uma situação rara ou extrema, muito mais comum no âmbito da saúde (Yin, 2015).

O estudo foi realizado em unidades oncológicas de duas instituições hospitalares de ensino do Brasil e os participantes foram enfermeiros que atuam nesse setor. O critério de inclusão da pesquisa foi enfermeiros que estivessem atuando há mais de um ano na área de enfermagem oncológica nas unidades selecionadas. Da mesma forma, os participantes que não responderam ao critério 
anteriormente citado, bem como, os que desistiram de participar da pesquisa, foram excluídos do estudo. Com o intuito de resguardar o anonimato, foram atribuídas letras E (N) a cada participante da seguinte forma: E refere uma forma simplificada para enfermeiro (tanto para os profissionais do sexo masculino quanto para o feminino) e $(\mathrm{N})$ refere-se a um algarismo cardinal que representa a ordem aleatória da realização da entrevista. A coleta de dados foi feita por meio de entrevistas semiestruturadas com 30 participantes, gravada no período de julho a setembro de 2014.

Cabe destacar que na pesquisa qualitativa buscam-se os fundamentos das situações vivenciadas. Assim, nesta abordagem, o número de enfermeiros entrevistados foi relevante devido à experiência destes em relação ao fenômeno investigado.

Neste estudo os dados foram analisados utilizando-se da abordagem qualitativa. O corpus da análise foi composto pelo material transcrito de gravações. Os dados foram organizados em subcategorias reagrupadas no grupo temático Educação Permanente e enfermeiros de unidades hospitalares oncológicas. O método utilizado para análise dos dados foi a Análise Temática, que consiste na descoberta de núcleos de sentido que compõem uma comunicação, cuja presença ou frequência signifiquem alguma coisa para o objeto analítico visado. Este tipo de análise se divide em três etapas: pré-análise; exploração do material, a categorização e tratamento dos resultados obtidos (Minayo, 2014). O trabalho foi desenvolvido de modo a garantir o cumprimento dos preceitos da Resolução 466/12 sobre pesquisa envolvendo seres humanos (Ministério da Saúde, 2012) e a coleta dos dados deu-se após a aprovação pelo Comitê de Ética em Pesquisa da Escola de Enfermagem de Ribeirão Preto da Universidade de São Paulo com o parecer $n^{0}$ 447. 978, bem como, a autorização pelas instituições, cenários do estudo.

\section{RESULTADOS e DISCUSSÕES}

Os resultados agrupados no núcleo temático Educação Permanente e enfermeiros de unidades oncológicas foram apresentados em duas categorias: Formação Acadêmica em Educação Permanente e Estratégias de Educação Permanente Organizacional e Individuais. Além disso, os resultados possibilitaram a identificação de uma categoria relacionada aos desafios enfrentados pelos enfermeiros.

\section{Educação Permanente e enfermeiros de unidades oncológicas}

\section{Formação Acadêmica em Educação Permanente}

Os resultados das entrevistas mostram que a formação acadêmica em Educação Permanente dos enfermeiros do estudo foi insuficiente para atuar em unidades oncológicas no âmbito hospitalar, pois, conforme os discursos observaram-se enfermeiros com preparo básico para desenvolver atividades de educação permanente, com disciplinas predominantemente teóricas e com enfoque na Atenção Primária à Saúde (APS).

Cabe ressaltar que todos os participantes relataram não ter tido o conteúdo de Educação Permanente, específico para atuar em clínicas especializadas, como a oncologia.

"Na graduação desenvolvemos educação permanente na Atenção Básica, não relacionado ao meu trabalho hoje, por exemplo, Diabetes, Cuidados com o bebê, em uma unidade básica de saúde, agora na área hospitalar nada, em oncologia menos ainda". (E04)

"Tive o tema educação permanente na disciplina de Administração na Atenção Básica, mas só o que era, mas focado na assistência especializada em oncologia não tive”. (E11)

O cenário de atenção a pacientes oncológicos apresenta um grande desafio para a transformação das práticas gerenciais e assistenciais em saúde, sob a ótica do enfrentamento do câncer de forma integral, em direção aos princípios do Sistema Único de Saúde (SUS).

Acredita-se que a formação do enfermeiro para prestar assistência ao paciente oncológico, é fragilizada, decorrente a lacunas 
encontradas no currículo generalista preconizado nas instituições de ensino. Estas instituições, por sua vez, devem-se responsabilizar pelo desenvolvimento da competência profissional, uma vez que o déficit de conhecimento dificulta que este profissional, ainda em formação em nível de graduação, possa se identificar com a área da oncologia, onde há a necessidade social de atuação competente dos diversos profissionais da área da saúde, considerando a incidência atual da doença (Luz, Vargas, Rosa \& Schmitt, 2016). O fato do conteúdo de educação permanente ter sido aprendido no Curso de Graduação em Enfermagem, predominantemente nas disciplinas da APS, se deve a portaria n. 198 de 13 de fevereiro de 2004 (Brasil, 2004), que institui no seu art.1 a Política Nacional de Educação Permanente em Saúde como estratégia do Sistema Único de Saúde para a formação e o desenvolvimento de trabalhadores para o setor. Em 2007 é implementada a portaria n.1996/2007 (Brasil, 2007) que estabelece novas diretrizes e estratégias, de modo a adequá-las às diretrizes operacionais e ao regulamento do Pacto pela Saúde (Brasil, 2006), que define a política de recursos humanos para o SUS, investindo na formação em serviço para os trabalhadores.O conteúdo de educação permanente obtido na formação acadêmica parece ser bastante limitado e pontual, dificultando dessa forma o desenvolvimento dessa competência para a atuação desses profissionais.

Especificamente em unidades oncológicas, a complexidade da clientela atendida leva a necessidade do aprimoramento e implementação de um grande número de atividades educativas para a promoção de um cuidado eficiente. Entretanto, cabe ressaltar que os discursos mostram o despreparo sobre a temática de educação permanente por parte dos enfermeiros desta área. Nesse sentido, as instituições empregadoras têm o papel de identificar as competências necessárias aos profissionais e promover estratégias para desenvolvê-las.

\section{Estratégias de Educação Permanente Organizacionais e Individuais}

No processo de múltiplas determinações e mudanças das práticas de enfermagem evidencia-se o papel fundamental das instituições de saúde para o desenvolvimento profissional dos enfermeiros. A educação permanente é uma estratégia gerencial fundamentada na percepção de educação como transformação das práticas assistenciais e aprendizagem significativa, centrada no cotidiano do processo de trabalho, na valorização do trabalho como fonte de conhecimento, com estratégias de ensino contextualizadas e participativas (Peduzzi, Guerra, Braga, Lucena \& Silva, 2009; Montanha \& Peduzzi, 2010), que devem ocorrer no espaço de trabalho como um processo dinâmico e contínuo (Barreto et al., 2013). Porém, os serviços de saúde, em especial as instituições hospitalares, buscam por quantitativo de pessoal de enfermagem centrado em procedimentos, atendimento das demandas e atividades de atualização pontuais.

"A gente costuma ter reuniões a cada dois meses com os enfermeiros para discutir os problemas aqui dentro da unidade, para depois realizar um treinamento específico, mas a gente ainda está caminhando nisso”. (E01)

"A gente sempre faz aulas para as meninas no computador, vai surgindo dúvidas a gente pesquisa junto, a gente aproveita a rotina, fora os treinamentos que damos para os funcionários”. (E19)

É importante destacar que as entrevistas explicitam ações que se aproximam da educação continuada, sendo que os enfermeiros priorizam atividades assistenciais estabelecidas pela rotina da instituição hospitalar, não visualizando o aprendizado contínuo e integral, conforme a concepção de educação permanente, que é pautada na proposta da aprendizagem significativa na unidade, onde, cada membro da equipe de enfermagem é o corresponsável pela assistência. Tendo em vista a abordagem da Educação Permanente quanto à perspectiva do cotidiano de trabalho, torna-se relevante identificar as atividades educativas oferecidas pelas instituições hospitalares. Os resultados das entrevistas possibilitaram agrupar as atividades educativas oferecidas pelas instituições hospitalares em Treinamento admissional e Cursos de atualização sobre temas variados da clínica, como Saúde da pele; Infecção hospitalar; Risco químico, físico e biológico; Cuidados paliativos; Coleta de 
Gasometria Arterial, NR-32; Hipodermólise e Biossegurança por meio do método de aulas expositivas.

Os discursos dos participantes revelam que essas atividades educativas, em ambas as instituições, não oferecem formação profissional específica na área de oncologia, fazendo com que os profissionais que atuam nestas unidades oncológicas, busquem por outras instituições especializadas a fim de suprir o seu desenvolvimento profissional quanto às competências necessárias para a sua atuação.

"O hospital não fornece recursos, os que têm são poucos... a gente fica muito limitado... a gente faz cursos e o treinamento admissional que abordam vários temas, agora curso em oncologia não tem nada, eu que fiz por fora, paguei do meu bolso”. (E02)

"A instituição oferece cursos de atualização voltados para enfermeiro generalista, não oferecem cursos de oncologia específico da clínica...a capacitação na área de oncologia é bem falho". (E30)

Os discursos mostram a necessidade das instituições hospitalares de ensino investir mais em cursos de atualização e formação profissional em unidades especializadas, especificamente na área de oncologia, pois este setor contém avanços tecnológicos e procedimentos avançados, tornando necessárias estratégias institucionais de formação em serviço, que favoreça segurança, eficiência e eficácia no planejamento do cuidado ao paciente oncológico. Com relação às atividades de educação em serviço realizadas pelos enfermeiros e sua equipe nas unidades oncológicas, destaca-se o ensino prático a partir da rotina da unidade, reuniões mensais para discutir a prática clínica em oncologia, e aulas para a discussão de casos com a equipe.

O ensino prático a partir das rotinas do serviço é de competência do enfermeiro. Portanto, torna-se imprescindível refletir sobre o papel do enfermeiro, enquanto líder da equipe de enfermagem, na promoção de condições para que possa desenvolver a supervisão com conhecimento e habilidades em unidades especializadas, caracterizando assistência conjunta com os outros membros da equipe a partir da reflexão crítica da realidade no setor. $\mathrm{O}$ envolvimento do enfermeiro no processo de educação permanente possibilita a este profissional se conscientizar sobre o seu papel e sobre a sua importância para aquele serviço, gerando mudanças qualitativas no seu processo de trabalho (Rossetti, 2015). O investimento nos trabalhadores possibilita que estes estejam preparados para atuar com segurança, responsabilidade e participando das tomadas de decisões (Pereira, Barbosa \& Vernasque, 2014). As atividades educativas citadas pelos enfermeiros participantes do estudo tem característica esporádica, pois, os participantes revelam a dificuldade de realizar educação permanente em serviço, devido a grande demanda de atividades assistênciais no ambiente de trabalho, baixo quantitativo de pessoal de enfermagem, sobrecarregando o trabalho; logo, essas atividades ficam prejudicadas, conforme os discursos a seguir.

"As estratégias que utilizo são treinamentos teóricos e práticos, decorrentes da rotina... A maior dificuldade é conciliar o ensino com a grande demanda do serviço". (E06)

"A gente aproveita a rotina, surgem dúvidas durante o treinamento admissional, vou tirando e realizando os procedimentos junto. A disponibilidade de tempo e poucos funcionários são fatores limitantes para essa unidade”. (E26)

As instituições hospitalares de ensino necessitam refletir e mobilizar quanto à escassez, ou má distribuição dos recursos, sejam eles físicos, materiais, humanos ou financeiros, pois, interfere na execução da educação em saúde. Dentre eles, os enfermeiros salientaram a questão da inadequação de pessoal de enfermagem e a sobrecarga de trabalho, dificultando a realização de atividades educativas de atualização da equipe de enfermagem.

\section{Desafios enfretados pelos enfermeiros}

No processo de educação permanente o acompanhamento dos profissionais na realização de suas atividades é etapa fundamental para a construção do seu conhecimento. Pois, a educação no trabalho, reconhece formas de articulação entre as 
diferentes áreas do conhecimento para estabelecer relação entre teoria e prática com vistas a propor intervenções factíveis e partilhadas com os envolvidos (Fagundes, Rangel, Carneiro, Castro \& Gomes, 2016). A análise dos dados dos entrevistados possibilitou dizer que a forma como está organizado o trabalho do enfermeiro pode ser fator limitante para o desenvolvimento de atividades de educação permanente, pois dependendo da forma como estiver organizado o seu trabalho, este não poderá participar ativamente nas atividades de educação permamente junto a sua equipe.

Os participantes do estudo apontam que um dos principais fatores limitantes na implementação da Educação Permanente é o dimensionamento de pessoal inadequado e a falta de planejamento para a realização das atividades educativas.

"A maior dificuldade do enfermeiro é ser assistencial e ao mesmo tempo treinar funcionário, isso é muito ruim, então priorizo a assistência, pois o paciente não pode ficar sem cuidados, os treinos e educação da equipe ficam para segundos planos”. (E07)

"A gente quer melhorar para o serviço, fazer especialização e cursos, mas o serviço vem e tira da gente, para fazer algo preciso me desdobrar em mil porque não tem funcionário suficiente e a demanda é grande, isso desanima e a educação fica de lado”. (E12)

Quanto ao quantitativo de pessoal de enfermagem, as instituições hospitalares devem cumprir as normas da Resolução do COFEN nº189/96 e n²93/2004 (Cofen, 1996, s.p.; Cofen, 2004, s.p.) que estabelecem os parâmetros oficiais para o dimensionamento de pessoal de enfermagem, definindo o quantiqualitativo mínimo nos diferentes níveis de formação para a cobertura assistencial. Tais iniciativas contribuem com os enfermeiros da prática na busca de solução para os problemas cotidianos de adequação de pessoal em um contexto onde a demanda, na maioria das vezes, é maior do que a oferta de atendimento, implicando em riscos para os clientes (Magalhães, Riboldi \& Agnol, 2009).Em busca de solução para o problema organizacional é imprecindível as instituições melhorarem o quadro quantiqualitativo de funcionários, possibilitando maiores oportunidades nas escalas para o enfermeiro desenvolver as estratégias de educação permanente, uma vez que esses profissionais julgam importantes para a segurança e qualidade da assistência em unidades oncológicas. É de fundamental importância que as instituições verifiquem as necessidades dos profissionais de modo a disponibilizar educação permanente, pois a rotina muitas vezes os consome, mas é preciso investimentos e tempo para conhecimento em profundidade das bases conceituais que guiam a prática (Silva, Curty, Duarte \& Zepeda, 2014).O enfermeiro possui papel crucial para que o trabalho de toda equipe de enfermagem aconteça, e a fim de garantir qualidade na assistência, ele próprio ou a instituição deve adotar estratégias motivadoras, buscando o aprimoramento profissional dos trabalhadores envolvidos nesse setor (Santos, Camelo, Laus \& Leal, 2015).

\section{CONSIDERAÇÕES FINAIS}

Os resultados mostraram que as estratégias de educação fornecidas pelas instituições hospitalares de ensino no estudo são os treinamentos admissionais e cursos sobre assuntos variados, possibilitando pouco investimento na formação de profissionais enfermeiros que atuam em unidades especializadas como a oncologia.

Quanto às estratégias de educação desenvolvidas pelos enfermeiros oncológicos destaca-se o ensino prático a partir das rotinas do serviço. Pois, percebeu-se a dificuldade enfrentada pelos enfermeiros no planejamento de atividades assistenciais e as atividades de educação permanente junto à equipe de enfermagem decorrente a alta de demanda de pacientes e baixo quantitativos de fucionários no setor.

Entendemos a limitação de estudos desta natureza, entretanto os resultados do estudo deverão contribuir para que os enfermeiros reflitam sobre sua práxis considerando a integralidade do cuidado prestado ao paciente oncológico, definindo novas modalidades, mecanismos e instrumentos de formação em serviço. Assim, como as instituições devem favorecer a formação permanente destes profissionais utilizando estratégias de ensino atualizadas, inovadoras e especializadas para este setor. 


\section{REFERÊNCIAS}

Barreto, B.M.F., et al. (2013). Educação continuada/permanente como estratégia no gerenciamento de enfermagem no sistema único de saúde: uma revisão integrativa. J. res.: fundam. Care, 5(3), 85-93. Recuperado de http://www.seer.unirio.br/index.php/cuidadofund amental/article/viewFile/1748/pdf_821.

Brasil. Ministério da Saúde. Portaria no 198/GM, de 13 de fevereiro de 2004. (2004). Institui a Política Nacional de Educação Permanente em Saúde como estratégia do Sistema Único de Saúde para a formação e o desenvolvimento de trabalhadores para o setor e dá outras providências. Brasília: Ministério da Saúde. Recuperado

de http://bvsms.saude.gov.br/bvs/publicacoes/politic a_nacional_educacao_permanente_saude.pdf.

Brasil. Ministério da Saúde. Portaria no 1996/GM, de 20 de agosto de 2007. (2007). Institui a Política Nacional de Educação Permanente em Saúde como estratégia do Sistema Único de Saúde para a formação e o desenvolvimento de trabalhadores para o setor e dá outras providências. Brasília: Ministério da Saúde. Recuperado de http://bvsms.saude.gov.br/bvs/publicacoes/politic a_nacional_educacao_permanente_saude.pdf.

Brasil. Ministério da Saúde. Portaria nº. 399/GM de 22 de fevereiro de 2006. (2006). Institui as Diretrizes operacionais do Pacto pela Saúde. Brasília: Ministério da Saúde. Recuperado de http://bvsms.saude.gov.br/bvs/publicacoes/prtG M399_20060222.pdf.

Ceccim, R.B. (2005). Educação pemanente em saúde: desafio ambicioso e necessário. InterfaceComunic., Saude, Educ, 9(16), 161-77. Recuperado de http://www.scielosp.org/pdf/icse/v9n16/v9n16a1 3.pdf.

Cofen- Conselho Federal de Enfermagem. (1996). Resolução Cofen $n^{\circ}$ 189/1996. Estabelece parâmetros para dimensionamento do quadro de profissionais de enfermagem nas instituições de saúde. Brasília: CFE. Recuperado de http://www.cofen.gov.br/resoluo-cofen-1891996revogada-pela-resoluo-cofen 2932004_4249.html.

Cofen- Conselho Federal de Enfermagem. (2004). Resolução Cofen $n^{\circ}$ 293/2004. Fixa e estabelece parâmetros para o dimensionamento do quadro de profissionais de enfermagem nas unidades assistenciais das instituições de saúde $e$ assemelhados. Brasília: CFE. Recuperado de http://www.cofen.gov.br/resoluo-cofen-1891996revogada-pela-resoluo-cofen2932004_4249.html.

Fagundes, N.C., Rangel, A.G.C., Carneiro, T.M., Castro, L.M.C., \& Gomes, B.S. (2016). Educação permanente em saúde no contexto do trabalho da enfermeira. Revista Enfermagem Uerj, 24(1), e11349. Recuperado de http://www.epublicacoes.uerj.br/index.php/enfer magemuerj/article/view/11349/17855.

Luz, K.R., Vargas, M.A.O., Rosa, L.M., \& Schmitt, P.H. (2016). Enfermeiros na atenção oncológica: conhecimento na prática do cuidado. Rev enferm UFPE on line, 10(9), 3369-76. Recuperado de http://www.revista.ufpe.br/revistaenfermagem/in dex.php/revista/article/view/8588/pdf_11016.

Magalhães, A.M.M., Riboldi, C.O., \& Agnol, C.M.D. (2009). Planejamento de recursos humanos de enfermagem: desafio para as lideranças. Rev Bras Enferm. 2009, 62(4), 608$12 . \quad$ Recuperado de http://www.scielo.br/pdf/reben/v62n4/20.pdf.

Minayo, M.C.S. (2014). O desafio do conhecimento: pesquisa qualitativa em saúde. São Paulo: Hucitec.

Ministério da Saúde. (2012). Resolução $N^{o}$ 466/2012. Diretrizes e normas regulamentadoras de pesquisas envolvendo seres humanos. Brasília: Ministério da Saúde. Recuperado de http://conselho.saude.gov.br/resolucoes/2012/Re so466.pdf.

Montanha, D., \& Peduzzi, M. (2010). Educação permanente em enfermagem: levantamento de necessidades e resultados esperados segundo a concepção dos trabalhadores. Rev Esc Enferm USP, 44(3), 597-604. Recuperado de http://www.scielo.br/pdf/reeusp/v44n3/07.pdf.

Nascimento, L.K.A.S., et al. (2012). Sistematização da assistência de enfermagem a pacientes oncológicos: uma revisão integrativa da literatura. Rev. Gaúcha Enferm, 33(1), 17785.

Peduzzi, M., Guerra, D.A.D., Braga, C.P. Lucena, F.S., \& Silva, J.A.M. (2009). Atividades educativas de trabalhadores na atenção primária: concepções de educação permanente e de educação continuada em saúde presentes no 
cotidiano de Unidades Básicas de Saúde em São Paulo. Interface (Botucatu), 13(30), 121-134. Recuperado

http://www.scielo.br/pdf/icse/v13n30/v13n30a11 .pdf.

Pereira, F.M., Barbosa, V.B.A., \& Vernasque, J.R.S. (2014). A experiência da educação permanente como estratégia de gestão com os auxiliares de enfermagem. Rev Min Enferm, 18(1), 228-235. Recuperado de http://www.reme.org.br/artigo/detalhes/921.

Rossetti, L.T. (2015). Educação permanente como ferramenta de gestão do Sistema Único de Saúde: a concepção dos enfermeiros. (Dissertação Mestrado). Escola de Enfermagem. Universidade de Juiz de Fora. Juiz de Fora.

Salimena, A.M.O., Teixeira, S.R., Amorim, T.V., Paiva, A.C.P.C., \& Melo, M.C.S.C. (2013). O vivido dos enfermeiros no cuidado ao paciente oncológico. Cogitare Enfermagem, 18(1), 142147. Recuperado de http://revistas.ufpr.br/cogitare/article/view/31320 /20027.

Santos, F.C., Camelo. S.H.H., Laus, A.M., \& Leal, L.A. (2015). El enfermero de unidades hospitalarias oncológicas: perfil y capacitación professional. Enfermería Global, 38, 301-3012. Recuperado de http://revistas.um.es/eglobal/article/view/190061/ 174331.

Silva, M.M., Curty, B.I.C., Duarte, S.C.M., \& Zepeda, K.G.M. (2014). Gestão de segurança de enfermagem em enfermarias de oncohematologia pediátrica. Rev Rene, 15(6), 91524. Silva, M.C. (2015). Atenção Oncológica do Colo de Útero no Brasil: as Políticas de Educação à Distância na Educação Permanente Profissional. UNOPAR Cient., Ciênc. Human. Educ, 16(3), 234-44.

Yin, R.K. (2015). Estudo de caso: Planejamento e métodos. Porto Alegre: Bookman.

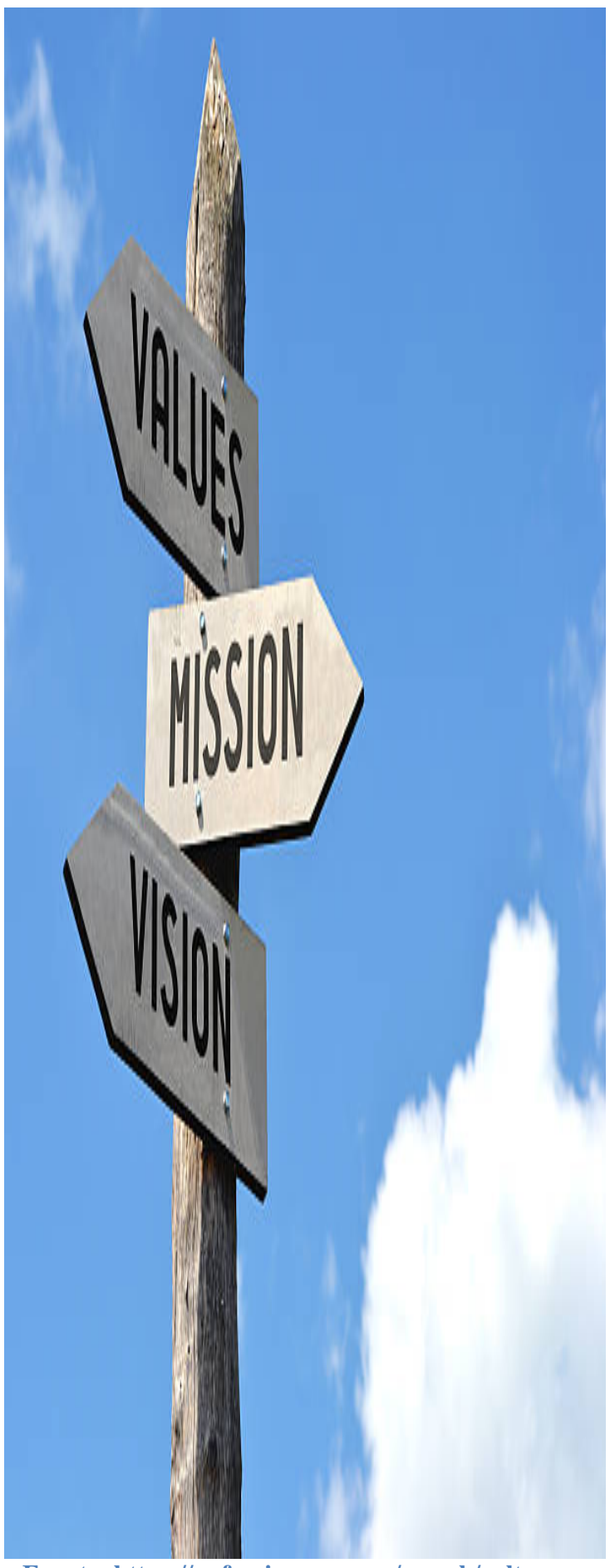

Fuente: https://es.freeimages.com/search/culture 